\title{
A Convolutional Neural Network(CNN) Classification To Identify The Presence of Pores in Powder Bed Fusion Images
}

Muhammad Ayub Ansari ( $\square$ muhammadayub.ansari@hud.ac.uk)

University of Huddersfield https://orcid.org/0000-0002-4775-2686

Andrew Crampton

University of Huddersfield

Rebecca Garrard

University of Birmingham

Biao Cai

University of Birmingham

Moataz Attallah

University of Birmingham

\section{Research Article}

Keywords: Porosity, LPBF, Convolutional Neural Network, In-situ Monitoring, Machine Learning

Posted Date: November 10th, 2021

DOI: https://doi.org/10.21203/rs.3.rs-1017967/v1

License: (c) (1) This work is licensed under a Creative Commons Attribution 4.0 International License. Read Full License

Version of Record: A version of this preprint was published at The International Journal of Advanced Manufacturing Technology on March 28th, 2022. See the published version at https://doi.org/10.1007/s00170-022-08995-7. 


\title{
A Convolutional Neural Network(CNN) classification to identify the presence of pores in powder bed fusion images
}

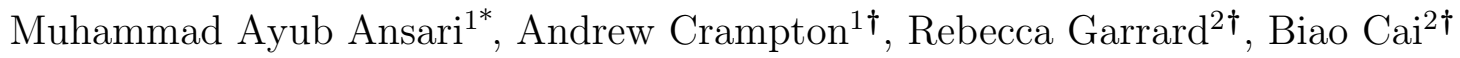 \\ and Moataz Attallah ${ }^{2 \dagger}$ \\ ${ }^{1 *}$ School of Computing and Engineering, University of Huddersfield, Queensgate, \\ Huddersfield, HD1 3DH, West Yorkshire, United Kingdome. \\ ${ }^{2}$ School of Metallurgy and Materials, University of Birmingham, , Birmingham, B15 2TT, \\ Edgbaston, United Kingdome.
}

\begin{abstract}
*Corresponding author(s). E-mail(s): muhammadayub.ansari@hud.ac.uk; Contributing authors: a.crampton@hud.ac.uk; R.H.Garrard@bham.ac.uk;

B.Cai@bham.ac.uk; M.M.Attallah@bham.ac.uk;

†These authors contributed equally to this work.
\end{abstract}

\begin{abstract}
This study focuses on the detection of seeded porosity during metal additive manufacturing by employing convolutional neural networks (CNN). The aim of the study is to demonstrate the application of Machine Learning (ML) in in-process monitoring. Laser Powder Bed Fusion (LPBF) is a selective laser melting technique used to build complex 3D parts. The current monitoring system in LPBF is inadequate to produce safety-critical parts due to the lack of automated processing of collected data. To assess the efficacy of applying ML to defect detection in LPBF by in-process images, a range of synthetic defects have been designed into cylindrical artefacts to mimic porosity occurring in different locations, shapes, and sizes. Empirical analysis has revealed insights into the importance of accurate labelling strategies required for data-driven solutions. Two labelling strategies based on the computer aided design (CAD) file and X-ray computed tomography (XCT) scan data was formulated. A novel CNN was trained from scratch and optimised by selecting the best values of an extensive range of hyper-parameters by employing Hyperband tuner. The accuracy of the model was $90 \%$ when trained using a CAD-assisted labelling, and $97 \%$ when using XCT-assisted labelling. The model successfully spotted pores as small as $0.2 \mathrm{~mm}$. Experiments revealed that balancing the data set improved the model's precision from $89 \%$ to $97 \%$ and recall from $85 \%$ to $97 \%$ when compared to training on an imbalanced data set. We strongly believed that the proposed model would significantly reduce post-processing cost and provide a better base model network for transfer learning of future ML models aimed at LPBF micro-defects detection.
\end{abstract}

Keywords: Porosity, LPBF, Convolutional Neural Network, In-situ Monitoring, Machine Learning

\section{Introduction}

Additive Manufacturing (AM) is a group of computer-controlled processes where threedimensional objects are manufactured by 
depositing material layer by layer [1]. AM, also known as 3D printing, is the backbone of Industry 4.0. Automotive, defence, aerospace, healthcare, and general manufacturing are some of the prominent areas where additive manufacturing is replacing conventional manufacturing [1]. The reduction of manufacturing costs and time, improved rapid prototyping, geometrical independence, rapid repair, and an ability to produce complex geometries using more sophisticated designs, are the main strengths of AM. Moreover, the weight of the object can often be reduced if produced using AM [2]. This is significant, particularly in the aerospace industry, as the reduced weight contributes to reduced oil consumption and carbon dioxide $\left(\mathrm{CO}_{2}\right)$ emissions [3]. According to an estimate by [4], by 2025, AM will reduce global manufacturing costs by 170-593 billion US dollars, with a 2.54-9.30 exajoules reduction in energy and 130.5-525.5 million tonnes (Mt) reduction of $\mathrm{CO}_{2}$ emissions.

Laser Powder Bed Fusion (LPBF) is a manufacturing technique where complicated geometrical objects are produced by melting pre-defined regions, producing a solidification of the metal powder, layer after layer [5]. LPBF is the most recommended method for metal construction of objects in the powder bed fusion process category [6]. Typical defects in LPBF include incomplete fusion of powder particles, porosity, powder contamination [7], cracks, surface deformation, irregularities in powder re-coating, and balling [8]. Among these defects, porosity is the most frequent and difficult to detect. Porosity compromises mechanical properties such as fatigue life [9]. It is particularly challenging due to its small size as it is difficult to observe with the naked eye. Several processing parameters, such as laser power, powder morphology, layer thickness, scan strategy, scan speed, gas flow, and hatch spacing can either directly or indirectly contribute towards the creation of porosity [8]. Incomplete fusion holes, voids, and keyholes are the main types of porosity. Incomplete fusion is caused by partial melting of the powder layer due to insufficient laser power [10]. This results in the melted layer failing to merge with the layer below, causing porosity [11]. Keyhole pores are caused by a high-energy input which vaporises the powder and leaves gas bubbles in the solidified metal [12]. Voids, on the other hand, are caused by rapid cooling which increases the residual stress of the melt pool [13]. The high value of laser powder and scan speed does not provide enough cooling time to melt-pool, resulted in voids.

The size and shape of the pores varies between different types of the porosity. Keyhole pores are round/spherical in shape and much smaller in size; typically greater than 50um [14-17]. The gaps created due to a lack of fusion are irregular, elongated and narrow in shape and usually greater than $200 \mu \mathrm{m}$ in size $[14,16,17]$. The largest pore size observed by Zhang et al. [18] was 340 $\mu \mathrm{m}$. Here, the pores were irregular in shape and could have been the result of a cluster of smaller pores. There were numerous factors that directly and indirectly influenced porosity. Increasing the energy density caused small, round pores (50$110 \mu \mathrm{m})$, whereas decreasing it below the optimum value resulted in pores as large as 250um [19]. $\mathrm{du}$ Plessis [20] stated that at higher laser power, the keyhole pores increased in number and size

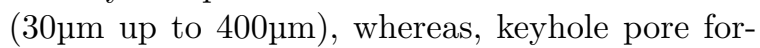
mation decreased at higher scan speeds. Choo et al. [21] studied the effect of varying laser power from $50-150 \%$. The maximum reported pore size was $340 \mu \mathrm{m}$. Leung et al. [22] categorised porosity into two types; gas pores and pores near the oxide layers. Gas pores observed in the experiments were in the range of $250 \mu \mathrm{m}$, whereas pores near the oxide layer merged with gas pores and grew as large as 50 to 500um. Mireles, Terrazas, Gaytan, Roberson and Wicker [23] had presented an image based close loop control of Electron Beam Melting (EBM) process. The artificial spherical pores of size $600 \mu \mathrm{m}$ to $900 \mu \mathrm{m}$ were created in test cylinders and were successfully detected from powder bed images. Mireles, Ridwan, Morton, Hinojos and Wicker [24] had designed spherical, triangular, cylinder and cubic shaped pores of $100 \mu \mathrm{m}$ to $2000 \mathrm{um}$ in the test $3 \mathrm{D}$ specimens. The experiments revealed that re-melting the porous layer reduced porosity.

Real-time identification of porosity is complex and difficult. Porosity can be detected using post-build evaluation techniques. However, post-build analysis can be expensive, time consuming, and laborious [25]. Several destructive (microscopic cross-sectional analysis) and nondestructive (Archimedes density measurement, gas pycnometry and XCT) methods are used to 
detect porosity [26]. Destructive methods, such as microscopic cross-sectional analysis, slice the 3D object to identify defects. However, this results in extra cost, time, and effort as well as wasted material.

The broader acceptance of AM technologies, especially in aerospace and the medical domain, is hindered by the lack of in-situ defect detection; as quality assurance is essential in these fields [27]. The main challenges to in-situ monitoring are limited view of the build chamber, poor spatial resolution of cameras, high temporal load, and the enormous amount of data collected [28]. Machine learning (ML) models are data-driven and known for their efficient and effective handling of large data sets. However, applications of ML in LPBF are relatively new and several issues are limiting the performance of ML solutions. The absence of publicly available data sets, the high cost of data capture, the installation of sensors, and data labelling are big challenges to solve for ML applications to be effective in in-process monitoring of the LPBF process [29]. Moreover, the scarcity of data, lack of experience in labelling data, lack of expertise in selecting good features and the issue of over-fitting and under-fitting of the derived ML models are also hindering the applicability of ML solutions in LPBF [30].

The aim of this research is to develop a ML model capable of identifying seeded defects in layer images from a LPBF build. The study aims to resolve the issues hindering ML applications in AM such as data capture, data labelling, extracting useful features from the data, class imbalance and over and under fitting of the ML models. A further aim is to demonstrate the effective hyper-parameter tuning could be used to produce an efficient model from the scratch without the need for transfer learning. We constructed 3D metal test specimens with rich porosity defects to acquire data for experiments. Seeded defects were used to artificially simulate porosity. A range of pore sizes was used to assess the ability of the LPBF to produce the pores and to identify any limits associated with the smallest detectable pore in the layer images captured during the build process. Three cylinders were designed containing seeded porosity. The porosity is inserted into the cylinders at a range of locations, with different shapes and dimensions. Seeded defects were used as it is difficult to create porosity in a controlled manner, especially when building the objects with several other objects in the same printing job. It is also difficult to identify the pores on images due to their small sizes. Using seeded porosity accomplishes two main objectives:

1. It provides us with a rich, synthetic data set able to mimic a range of pore sizes and types to allow detectable defects to be studied.

2. It provides geometrical information to assist in labelling of the image data captured during the build process.

This helps in data labelling as the exact location of the porosity in the powder bed images from the CAD file is known; reducing the need for experts in the labelling stage significantly, as well as avoiding the need to use expensive, time-consuming destructive methods. Two labelling approaches for the image data set were devised:

1. The image data set labelled according to the CAD design information.

2. The same image set labelled with the help of post-build XCT scans.

The key intuition here is that the seeded CAD information helped to build a valid and reliable model and the XCT showed how we could use non-destructive testing on the $3 \mathrm{D}$ samples to tune the model for non-seeded applications. The nondestructive XCT scans of the test cylinders were obtained and correlated with the in-process build images. A full analysis was performed to evaluate the ability of the ML model to accurately detect different types and size of pores.

A deep convolutional neural network (CNN) was trained using the in-process images. A CNN approach was chosen due to its superior ability to automatically extract features from the images without the need for human interaction. Manually extracting useful features from images is extremely difficult [30] and can often fail to capture local, spatially-related information. Convolutional neural networks combine advanced image processing techniques with deep neural networks. This makes CNNs extremely powerful and computationally more efficient when compared to the other common ML models such as Decision Trees [31], Random Forests [32], and Support Vector Machines (SVMs) [33] etc. In a comparison by Chouiekh and EL Haj [34], CNNs outperformed traditional ML models such as SVMs, Random 
Forests and Gradient Boosting Classifiers, both in terms of accuracy and training time. CNNs are scalable and capable of handling big data. Another significant advantage of CNNs over traditional ML classifiers is their ability to incorporate transfer learning, allowing complex models to be trained efficiently for a wide range of applications. Transfer learning is the process of keeping the weights and learning of a trained model and using it to solve new similar problems with little re-training $[35,36]$. Going forward, the authors intend to use transfer learning on the current models to identify different defect types. This will allow the learned weights of the current CNN model to be retained and transferred to new, possibly more complex, deep CNN models to identify a wider range of defects.

The remaining sections of the paper are organised as follows: in section two, related work is discussed. Section three details the materials used in the experiments, the chosen model structure, and explains the experimental methodology in more detail. Results and discussion are presented in section four and the conclusions are presented in section five.

\section{Related Work}

Porosity detection from images captured during printing is a highly studied defect in AM. However, identifying the existence of porosity in LPBF parts is difficult and different researchers have followed different experimental approaches. Capturing the porosity on images is challenging and requires a high resolution camera and a well-lit build chamber. Mireles, Ridwan, Morton, Hinojos and Wicker [24] designed pores of size in range of 100 to $2000 \mu \mathrm{m}$ and of various shapes (sphere, cubes, circular, triangular and prism) in their test specimens. HIPing and re-scanning were used to reduced the porosity. CT scanning performed $60 \%$ better compared to IR camera in recording porosity on images. Moreover, IR camera used were unable to detect pores smaller than $600 \mu$. Similarly, Mireles, Terrazas, Gaytan, Roberson and Wicker [23] had created artificial seeded spherical pores of size $600 \mu \mathrm{m}$ to $900 \mu \mathrm{m}$ in test cylinders. Other researchers have created 3D metal objects with porosity induced by varying the laser power, scan speed, and hatch spacing [25, 37-39]. ML algorithms are being widely used for defect detection in LPBF with varying degrees of success. Many studies have used different ML algorithms to identify porosity from powder bed images. The most prominent of those studies are discussed here.

One of the prominent work that developed a ML model using images from the build chamber was carried out by Aminzadeh and Kurfess [40]. They employed a Bayesian classifier to detect defects from images. The proposed framework achieved a precision of $89.5 \%$. Gobert et al. [25] aimed to identify porosity types (gas pores and elongation voids) using images from the build chamber and from XCT scan data, labelled by human experts. The layer-wise images were classified as nominal or flawed using a binary linear support vector machine (SVM) with a high accuracy of $85 \%$. A similar effort by Kwon et al. [37] identified porosity by using 13,200 images from the build chamber. The experiments were divided into seven different groups based on different laser power ranges from $50 \mathrm{~W}$ to $350 \mathrm{~W}$, keeping the rest of the printing parameters constant. The experiments revealed that laser power less than 250W caused porosity. The trained neural network identified porosity images with less than a $1.1 \%$ failure rate. Zhang et al. [38] trained a CNN model on images of a 3D metal object printed with titanium powder using direct laser disposition. Five specimens of length $15 \mathrm{~mm}$ each were printed with varying scan speeds of $1-4 \mathrm{~mm} / \mathrm{s}$ and laser power of 150-250W. Both destructive, crosssectional analysis and non-destructive (XCT scan) was performed to identify the porosity location in the test objects. The model achieved an accuracy of $91.2 \%$ for micro pores as small as $100 \mu \mathrm{m}$ [38].

In-situ melt pool monitoring is another highly studied area. Many defects, such as keyhole porosity, spatter, balling, and under-melting occur around the melt pool. Many studies have focused on melt pool monitoring to investigate the causes and identification of defects. Yuan et al. [29] proposed a semi-supervised CNN for SLM process monitoring. 1,200 individual tracks of $5 \mathrm{~mm}$ were printed using $316 \mathrm{~L}$ stainless steel powder. The data set consisted of frames of size 250x250 pixels extracted from 1,200 LPBF videos, out of which 700 were labelled manually. The experiments showed that a semi-supervised approach achieved an accuracy of $93.8 \%$ compared to $92.2 \%$ 
experimental scenarios were designed by varying scan speed 50-500mm/s and laser power 50-150W. The proposed DBN identified five different melted states with an $83.4 \%$ accuracy. Moreover, the proposed framework required less parameters, feature extraction and signal processing [45].

An in-situ, bi-stream, deep convolution neural network, which aimed to identify insufficient layer densification, trace discontinuity and surface deformation defects in LPBF, successfully identified the process-induced errors with an accuracy of 99.4\% [46]. Scime and Beuth [47] aimed to identify six anomalies in LPBF; recoater hopping, recoater streaking, debris, super elevation, part damage and incomplete spreading. Previously, Bag of Words (BoW) and a CNN were used for multiobject detection from a single image. The BoW technique relies heavily on human input. The authors proposed a Multi-scale CNN (MsCNN) based on reinforcement learning of the AlexNet CNN which was trained on a colour image data set called ImageNet. The images were captured from 53 builds (of 3D printed objects) on an EOS M290 LPBF machine. The training data set was composed on 10,071 multi-scale patches in total, out of which 3,827 were defect-less, 1,896 recoater hopping, 527 recoater streaking, 666 super elevation, 1,297 disturbance and 1,858 incomplete spreading patches manually labelled by human experts. The proposed MsCNN outperformed the BoW and CNN, and was less effected by human bias [47]. In a similar effort to identify powder anomalies, by the same authors, a computer vision-based solution was proposed that has the capability to be used as a real-time control model with some sufficient future improvements [48]. The image data set of 2,402 images, out of which 1,040 were faultfree images, 264 recoater hopping patches, 228 recoater streaking patches, 187 debris, 314 super elevation, 264 part failure and 105 incomplete spreading patches were captured from an EOS M290 LPBF machine using only the built-in camera and LED light. The proposed computer vision algorithm successfully identified failure modes and the exact location of flaws in the final product with microscopic accuracy. However, the use of deep learning algorithms with further improvements in the accuracy of the ML models will be required to use it as an in-situ monitoring algorithm [48]. captured by a near-infrared camera. The test specimens were printed on a custom design SLM machine with an integrated infra red camera. Five 


\section{Materials and Methods}

The specimens for the experiments were constructed using LPBF. A diagram of a typical LPBF process is shown in Fig 1. A dose of metal powder is spread uniformly over the build plate by a wiper or recoater system. A laser (or lasers) is directed to melt specific regions on the newly spread metal powder layer. 3D objects are formed by successively melting $2 \mathrm{D}$ cross-sections of the whole 3D object. After each layer is melted, the powder bed is lowered by the thickness of the powder layer, and a new powder layer is spread. To avoid oxidation of metal during the melting process, the oxygen level inside the chamber is kept to a minimum by introducing inert gases such as argon or nitrogen.

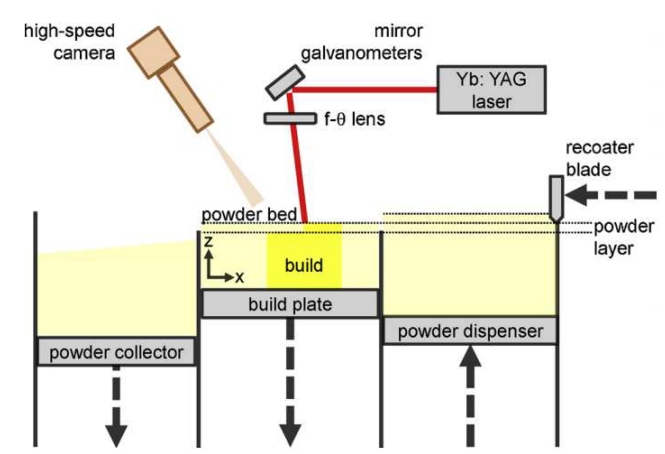

Fig. 1 Illustration of the LPBF Process [42]

A sample geometry was designed to incorporate seeded defects of different sizes to explore the minimum pore size that can be created by the LPBF machine by validation with XCT of the built parts, and to identify the smallest pore size that can be captured by the layer imaging camera. The pores were designed to range in size from $20 \mathrm{um}$ to $2 \mathrm{~mm}$, as shown in Fig 2. Different defect shapes were used (spherical and cubic) to see if this affected detection of the pores.

Three cylinders, each with a height of $30 \mathrm{~mm}$ and a diameter of $12 \mathrm{~mm}$, were designed to contain seeded pores within them. Two cylinders, B1 and B2, had circular pores whereas B3 had cubical pores. Similar seeded porosity experiments were performed by [24]. Spherical and cubic pores were selected here in order to study the application of deep neural networks in identifying defects from the images irrespective of the shape of the pores.

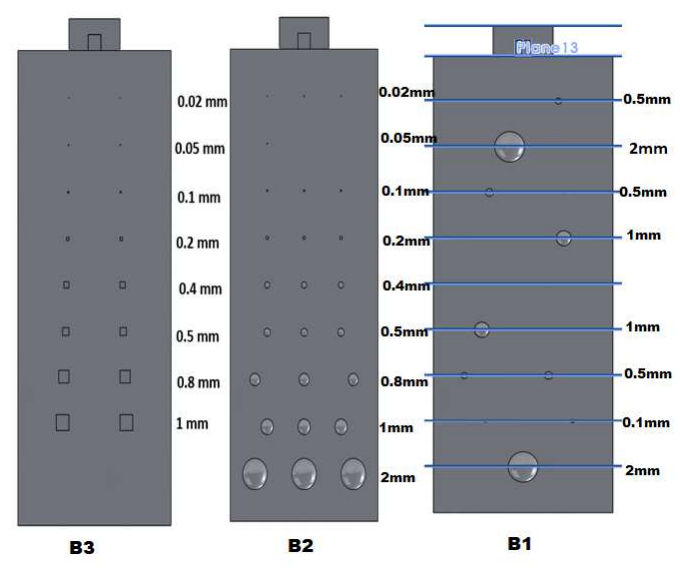

Fig. 2 Computer Aided Design of three cylinders

The cylinders were produced using an SLM500HL machine (SLM Solutions, Germany) from A20X powder with 20-53um size distribution. The cylinders were built on an aluminium substrate which was held at $150^{\circ} \mathrm{C}$ throughout the build process. The process parameters used were $360 \mathrm{~W}$ laser power, $1500 \mathrm{~mm} / \mathrm{s}$ scan speed, $100 \mu \mathrm{m}$ scan spacing, and a layer thickness of $30 \mu \mathrm{m}$. The stripe scan strategy was used.

The layer imaging camera used in this study is the LayerCam system in an SLM500HL machine. This comprises of two Baumer TXG20 cameras, capturing $2600 \times 1,440$ pixel images of the build area $(500 \times 280 \mathrm{~mm})$. This results in an approximate pixel size of $0.2 \mathrm{~mm} /$ pixel. The cameras are not centred on the build area. Image transformation operations are carried out by the SLM500HL machine, before the images are saved for the user. The capturing of layer images is automated by the SLM500HL machine. Flash strips inside the machine are triggered when capturing the images. An image is captured after each powder layer is spread, and after each layer is melted. Therefore, two images are captured per layer. The capturing of data in this manner on every layer of the build enables the examination of both the interior and exterior of the specimens. The images are saved with a timestamp and layer number in the file name.

\subsection{Data Acquisition}

After the build was completed, the layer images from the build chamber were processed prior to being passed to the ML models. It is common 
practise to build more than one object simultaneously in a LPBF machine to save time and money. This was the case when printing the cylinders as they were built with various other objects. The irrelevant portions of images were discarded through standard image processing and the regions of interest (ROI) containing the cylinders were extracted. The final images of each cylinder were 190 pixels in height and 150 pixels in width. An error with the camera system during the printing resulted in some missing layers in the image data set. Missing images and different starting and ending points for different cylinders were handled by renaming the cropped images. The new names consisted of: a porosity flag, cylinder name, layer number, and the size of pore. The final data set had 963 images for each cylinder.

\subsection{Data Labelling}

A supervised machine learning model requires labelled data for training. Labelling the images is a big challenge in $\mathrm{AM}$ and requires the input of domain experts. This is a laborious, time consuming and human intensive task. We designed the $3 \mathrm{D}$ objects with seeded pores with the purpose of overcoming this hurdle. Two approaches were formulated to label the images. For both of the approaches, experts in additive manufacturing supervised the labelling process.

Approach 1: CAD assisted labelling. The images from the printer were labelled based on the CAD file design. The $30 \mathrm{~mm}$ cylinders, built with uniform layer thickness of $30 \mu \mathrm{m}$, resulted in 1,000 images. Since the pores were designed in the CAD file, the location of the pores was known. A visual representation of the CAD designs, along with the pore sizes and shapes is shown in Fig 2.

Approach 2: XCT assisted labelling. We employed post-build XCT scans to assist with the image labelling. The XCT images are superior in terms of quality and are a reliable source to see whether the pores actually formed or not inside the final 3D object. Some of the layer images, along with their corresponding XCT images, are shown in Fig 3.

\subsection{Data Augmentation}

The data set consisted of 2889 images. However, the data was highly imbalanced. There were 2386 non-porosity images and only 503 images which

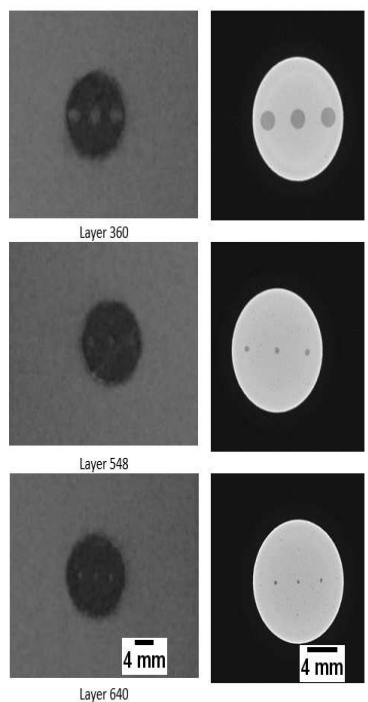

Fig. 3 Sample powder bed and their corresponding XCT images. The images on the left are from powder bed and on the right are their corresponding images from XCT analysis

\begin{tabular}{ll}
\hline Variable & Values \\
\hline Vertical Flip & True \\
Horizontal Flip & True \\
Width Shift Range & 0.1 \\
Height Shift Range & 0.1 \\
Fill Mode & Nearest
\end{tabular}

Table 1 Data Augmented parameters to over-sample the porosity images

contained porosity when using the CAD-assisted labelling approach. The class imbalance was even more significant in the XCT-assisted labelling approach, resulting in 2578 non-porosity and only 311 porosity images. Class imbalance is often a major hurdle in training an unbiased machine learning model - particularly when the minor class is the one we wish to accurately predict. To address this problem we employed data augmentation methods to over-sample the minority class; the porosity images. The data-augmented parameters used in the experiments are shown in Table 1 along with their values. The new, balanced data set consisted of 5135 total images of which 2578 were non-porosity and 2557 were porosity.

\subsection{Data Pre-processing}

The images captured from the SLM500HL (shown in Fig 3) contained a lot of noise. To improve the standard of the images, image thresholding 
was applied. The images in the grey-scale channel show two peaks in their pixel histogram analysis. Therefore, Otsu's Binarization [49] was applied to the images to improve their quality. Fig 4 shows a sample of porosity and non-porosity images after the Otsu thresholding. The images were re-scaled and their pixel values were normalised before feeding them into the CNN. The data set was split into

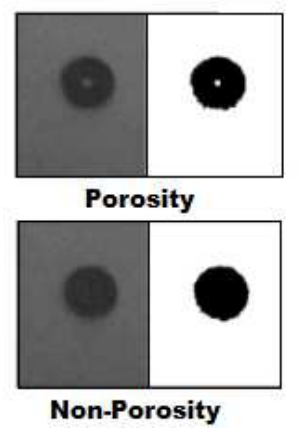

Fig. 4 Sample images showing porosity and non-porosity in a layer image. The left image is the layer image and the right image is the same image, binarized using Otsu thresholding [49]

$70 \%$ training and $30 \%$ testing using a stratified split to ensure the same class distribution in both train and test sets. The training data set had 1804 non-porosity and 1790 porosity images. Whereas, the test data set had 774 non-porosity and 767 porosity images.

\subsection{Hyper-parameter Tuning}

The convolutional neural network was selected to distinguish between porosity and non-porosity images. CNNs are known for their ability to extract features from images and are widely used in medical [50-52] and commercial applications $[53,54]$. However, selecting the CNN architecture and training it from scratch requires significant computational resources and time. Moreover, selecting the right values for the CNN's hyperparameters is crucial for its learning and predictive ability.

The goal is to design an effective CNN architecture with good predictive capability and in a reasonable training time. To find the best architecture we experimented with six different CNN architectures starting from a simple 1 convolutional layer, 1 max pooling layer and 1 dense layer

\begin{tabular}{cccc}
\hline Model & Conv Layers & Pooling Layers & Dense Layers \\
\hline $\mathbf{1}$ & 1 & 1 & 2 \\
$\mathbf{2}$ & 1 & 1 & 3 \\
$\mathbf{3}$ & 2 & 2 & 2 \\
$\mathbf{4}$ & 2 & 2 & 3 \\
$\mathbf{5}$ & 3 & 3 & 2 \\
$\mathbf{6}$ & 3 & 3 & 3 \\
\hline
\end{tabular}

Table 2 CNN Model's Architectures

\begin{tabular}{lll}
\hline & Hyper-parameters & Range \\
\hline \multirow{4}{*}{ Conv Layer } & Conv Filters & $32,48,64,80,96$ \\
& Conv Kernel & $3,5,7$ \\
Pooling Layer & Conv Activation & tanh, elu, relu \\
Dense Layer & Units & $2,4,6,8,10,12$ \\
Algorithm & Activation Function & $128,192,256,320,384,448,512$ \\
Lanh, elu, relu \\
Table 3 Hyper-parameters and their range of values for \\
fine-tuning the various models
\end{tabular}

to 3 convolutional layers, 3 max pooling layers and 2 dense layers. The architecture of the six models are shown in Table 2. Various hyper-parameters associated with each unique layer of the CNN were experimented with using a range of values. The hyper-parameters and their range of values used in the hyper-parameter tuning is shown in Table 3. We have employed ADAM for our experiments. ADAM is a well-known optimisation algorithm, both computationally and memory efficient, and known to perform well for big data problems [55]. Finding the best values for the hyper-parameters of a CNN model requires extensive, computational and memory resources. Commonly-used hyperparameter optimizers, such as random search and Bayesian optimizers, are slow and require significant amounts of memory. Hyperband uses early stopping criteria to speed up the hyper-parameter optimization and is known to be 5 to 30 times faster than Bayesian optimization methods [56]. We used the Hyperband optimization algorithm to find the best values of the hyper-parameters. The best performing values of the set of hyperparameters of each of the six CNN models are shown in Table 4 along with the accuracy and the loss value of the respective model. All of the model architectures achieved great accuracy and loss. We selected the architecture of model 3 and its respective hyper-parameters for our experiments. Model 3 achieved the best accuracy and loss score on the test data and had comparatively less total trainable model parameters, making it a more efficient model to evaluate. 
Table 4 CNN Models and their accuracy on the best hyper-parameter values

\subsection{Convolutional Neural Network Model}

The CNN is a powerful classifier with the ability to transfer its learning from one similar problem to another [57, 58]. The reason for not using a pretrained CNN model via transfer learning in this instance, is due to the uniqueness of the problem at hand. The well-known pre-trained CNN models such as VGG, ResNet, EfficientNet, Inception, 
etc., are mostly trained on thousands of ImageNet images. However, none of them have been trained on powder bed images, which are very different to the ImageNet images. Just to demonstrate the ineffectiveness of pre-trained CNN models, we downloaded VGG16 and trained it on powder bed images for porosity identification. The model was trained on a balanced data set. The model failed to identify a single porosity image and predicted all images as non-porosity. The model's accuracy was $52 \%$.

The novel CNN constructed for the experiments is shown in Fig 5. The values of the hyperparameters were selected based on the hyperparameter tuning results described in the previous section. The final model consists of two convolution layers followed by a max pooling layer after each convolution layer. Both convolutional layers had 96 filters, used 'same' as the padding method and had the kernel size set to 3 . However, the first convolutional layer used 'tanh' whereas the second used the 'relu' activation function. Two max pooling layers followed by each convolutional layer had a stride sliding window size of 4 . The "flattened layer" was followed by two dense layers with zero drop out. The first dense layer had 448 units and used the 'tanh' activation function. The final layer, utilising the soft-max activation function, had two neurons corresponding to the two possible outcomes: "porosity" or "non-porosity". The learning rate of 0.0001 was used for the ADAM optimization method. The model was trained using the categorical cross-entropy loss function.

\section{Results and Discussion}

A comparison of the results obtained by following both labelling approaches, CAD-assisted labelling and XCT-assisted labelling, are shown in Figs 6 and 7 . The model trained on images labelled using the CAD design achieved an accuracy of $90 \%$. Whereas, the model trained on the XCT-assisted labelling approach achieved $97 \%$ accuracy in identifying porosity images from non-porosity images.

Class imbalance is one of the most common challenges in data-driven solutions. Class imbalance occurs when there is a big difference in the number of occurrences of one class compared to other classes within the data set. When this is the case, accuracy, used as an evaluation metric, can be very misleading if the data is biased towards a particular class. In these experiments, more than $88 \%$ of the images were without porosity in the XCT assisted labelling approach, whereas in the CAD assisted labelling approach $83 \%$ of the images belonged to the non-porosity class. A model trained on imbalanced data would result in a biased model. The CNN model was trained on both balanced and imbalanced data set to study the impact of balanced and imbalanced data on the model's training. We used data augmentation methods to combat the class imbalance problem. The XCT-assisted labelled images were balanced by oversampling the minority class. The balanced XCT-assisted images had 2578 non-porosity (50.20\%) and 2557 (49.79\%) porosity images. The model's accuracy on balanced and imbalanced XCT assisted images was 97\%. However, it is always desirable to evaluate the model with different evaluation criteria instead of just relying solely on accuracy. Apart from precision and recall, the F1-measure was employed, which allows a harmonic mean to be used to correctly weight the accuracy measure to account for the imbalance. Further insight can additionally be obtained by considering the confusion matrix and further metrics that can be obtained from it. Precision of a binary classifier is given by:

$$
\text { Precision }=\frac{\text { TruePositive }}{\text { TruePositive }+ \text { FalsePositive }}
$$

Precision is the measure of true positives among all the predicted positive cases. In other words, it specifies how many among all predicted positive cases are actually true. Precision can prove to be an excellent metric for a manufacturer, where controlling false-positives is critical for the business. On the other hand, recall can prove to be a better indicator of the model's performance when false-negatives have a high impact. The recall is calculated as:

$$
\text { Recall }=\frac{\text { TruePositive }}{\text { TruePositive }+ \text { FalseNegative }}
$$

It is a measure of how many of true positives are actually identified. In binary classification with significant class imbalance, often, a critical task is to identify between the false-positive and falsenegative rates. For instance in cancer diagnostics, a false-negative is more dangerous compared to 
Fig. 6 Comparison of model evaluation metrics for both labelling approaches on non-porosity class

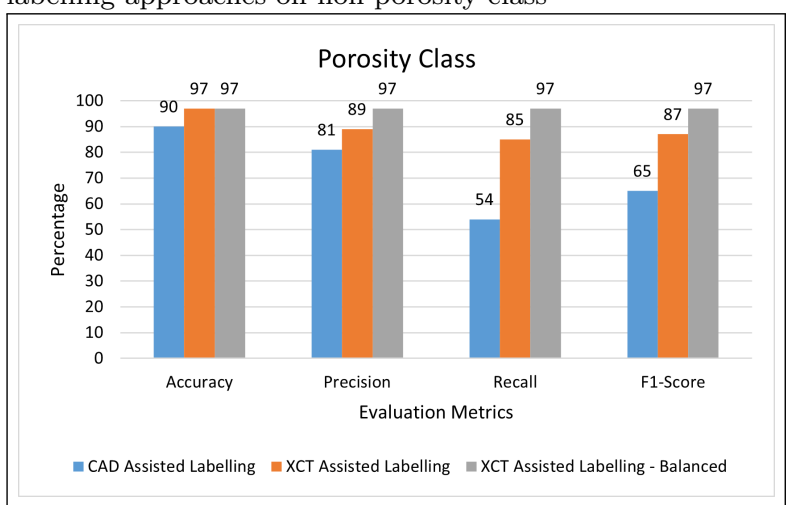

Fig. 7 Comparison of model evaluation metrics for both labelling approaches on porosity class

a false-positive. Similarly, in our case a falsenegative is more critical. An image with porosity identified as non-porosity is more dangerous as it will build the object with porosity. This demonstrates why recall is a better evaluation criteria for the model's performance. For non-porosity, the recall of the model trained on in-process
CAD-labelled images was $97 \%, 99 \%$ for imbalanced XCT-labelled images and $97 \%$ for balanced XCT-labelled images. However, for images with porosity, there is a significant difference in the recall of the two approaches. The recall on CADlabelled images was $54 \%$ while the recall of imbalanced XCT-labelled images was $85 \%$. Whereas, the recall of the model for balanced XCT-labelled images was $97 \%$. The models' precision, recall and F1-score on balanced XCT-labelled images was $97 \%, 97 \%$ and $97 \%$ respectively. Whereas the model's precision, recall and F1-score on imbalanced XCT-labelled images was $81 \%, 85 \%$ and $87 \%$ respectively. The balanced data set resulted in a better, more generalised and unbiased training of the model; as the model outperformed in terms of precision, recall and F1-score. The model performed well for the majority class, non-porosity images, and there was very little difference in terms of precision, recall and F1-score as shown in Fig 6.

The reason for a poor recall value for CADlabelled images was due to the high false-negative rate. This can be observed in Fig 8. The false-negative rate of the CAD-assisted labelling approach was $45.65 \%$. This means that out of all images with porosity, $45.64 \%$ was wrongly classified as non-porosity images by the model. This is a significantly high number of missclassifications of a crucial class. Whereas, the rate of false-negatives in the imbalanced XCT-assisted labelling approach was only $14.72 \%$ and $3.26 \%$ for balanced XCT-labelled images. This shows 
Fig. 8 Comparison of false-positive and false-negative results from CAD-assisted labelling and XCT-assisted labelling

that only a fraction of porosity images was missclassified by our model when trained on balanced XCT-labelled images. The better performance of $\mathrm{XCT}$-assisted labelling is mainly due to the better and more correct labelling of the image set. The CAD-assisted labelling had many non-porosity images wrongly labelled as porosity images. The false-positive rates of the model were insignificant; in the CAD-labelling approach the false-positive rate was $2.6 \%, 1.36 \%$ for imbalanced XCT-labelled and $2.84 \%$ for balanced XCT-labelled images. This means that both CAD and XCT labelling approaches worked well in predicting the nonporosity images and only a small percentage of non-porosity images were wrongly predicted as porosity images. A significant difference between the false negative $(14.72 \%)$ and the false positive rate $(1.36 \%)$ was observed for imbalanced XCTlabelled data. However, the false positive (2.84\%) and false negative $(3.26 \%)$ rates for balanced XCT-labelled images were very insignificant, and resulted in better, unbiased training of the model.

As stated previously, we chose spherical and cubical shapes for the seeded pores in our test cylinders. Experiments have revealed that the spherical and cubic pores have a similar appearance in the layer images. The model achieved an excellent accuracy of $97 \%$ on the image data set labelled with the help of XCT. The model's accuracy in predicting the different sized pores is shown in Fig 9. The model attained an accuracy of $95.53 \%$ on the largest sized pores; the $2 \mathrm{~mm}$. Similarly, the model predicted the $1 \mathrm{~mm}$, $0.8 \mathrm{~mm}$, and $0.5 \mathrm{~mm}$ sized pores with more than $80 \%$ accuracy. The smallest visible pore on inprocess images, using the current printing setup, was $0.2 \mathrm{~mm}$. Our model was $66.67 \%$ accurate in predicting the $0.2 \mathrm{~mm}$ sized pores. The model's ability to correctly identify pores reduced with the reduction in the pore sizes. A slightly better accuracy on $0.4 \mathrm{~mm}$ pores is due to the more instances of $0.4 \mathrm{~mm}$ pores in the data set compared to the other pore sizes. Apart from accuracy, precision,

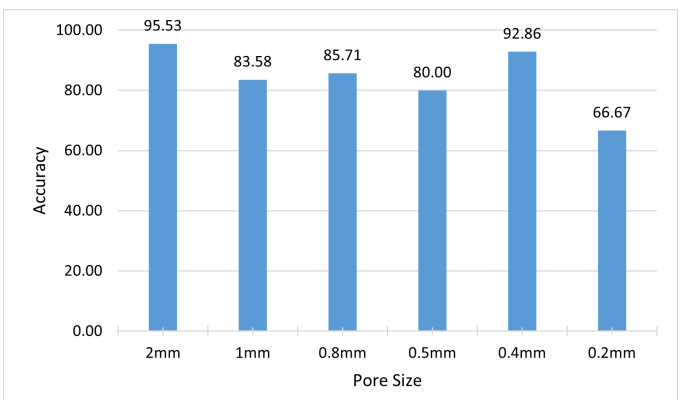

Fig. 9 Model accuracy for different pore sizes

recall and F1-score, the model's loss curves were also calculated to ensure the model's good training and generalisation. The loss curve of the final model trained on balanced XCT-labelled images is shown in Fig 10. The model is clearly overfitting as the train and test loss curves diverges after some initial epochs of training. We used $l_{2}$ kernel regularisation with regularisation factor of $l=0.01$ and $50 \%$ dropout to avoid the over-fitting of the model. The final model's loss curve with regularisation is shown in Fig 11.

\subsection{Critical Evaluation of Overall Results}

Porosity is a challenging defect in AM. It has been reported that certain porosity types are not detectable with image processing [43]. Due to the small size of pores, the layer imaging system of many LPBF printers fail to capture the porosity on the images. XCT analysis is a post-build technique known for its high resolution images. The images acquired by the XCT analysis of 3D metal objects are much clearer, cleaner, and of higher definition. Therefore, XCT scanning is better in identifying the small pore sizes compared to in-process imaging. [24] stated that XCT scanning is able to find approximately $60 \%$ of smaller sized pores when compared to an infrared imaging camera. 


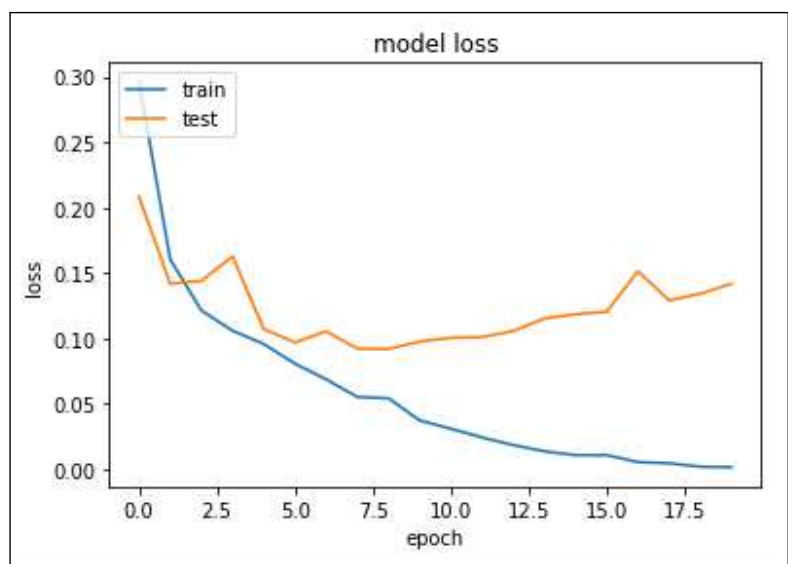

Fig. 10 Loss curve of final model with no regularisation

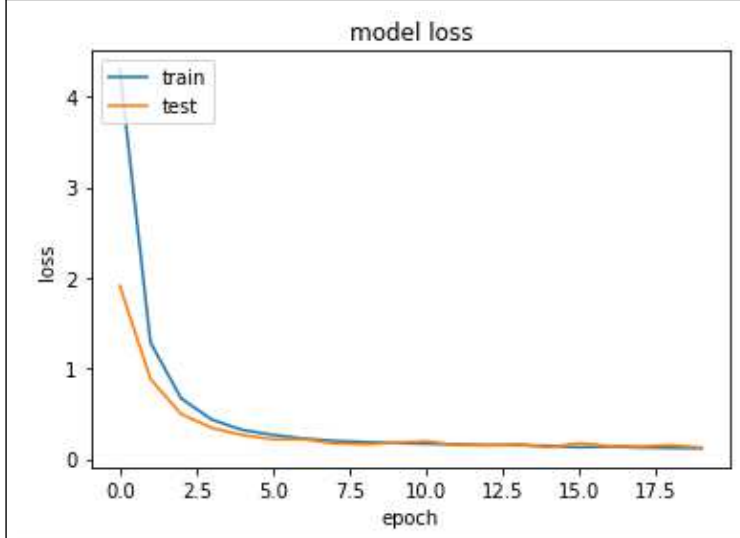

Fig. 11 Loss curve of final model with $l_{2}$ regularisation and dropout

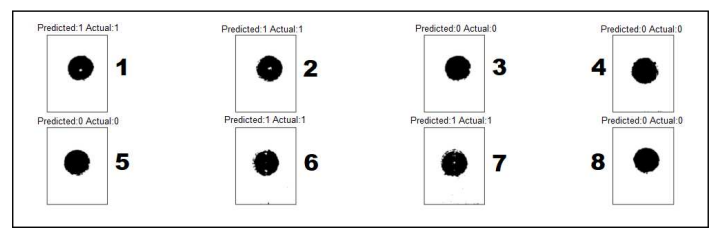

Fig. 12 Sample images of correct predictions of the CADlabelled images

The accuracy of the CAD labelling approach was lower than that of the XCT labelling approach due to incorrectly labelled images. The images were labelled according to the CAD design but some pores designed in the CAD file were not created in the final test specimen. This caused an incorrect labelling of some images as porosity images when they should have been labelled as non-porosity images. Some of the correctly predicted images are shown in Fig 12. The images numbered 1, 2, 6 and 7 in Fig 12 had porosity and were correctly predicted by the model. The

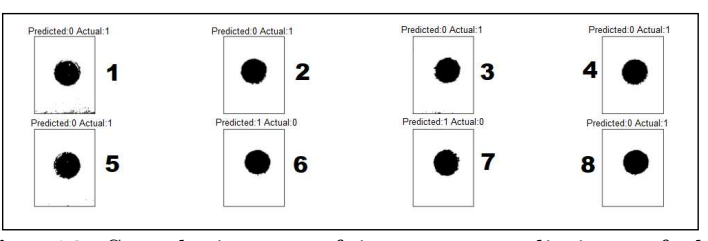

Fig. 13 Sample images of incorrect predictions of the CAD-labelled image model

remaining images $(3,4,5$ and 8$)$ were also correctly identified as non-porosity images. However, the model failed to classify some images correctly. Someone of the wrongly classified images are shown in Fig 13. The images numbered 2, 3, 4 and 8 in Fig 13 showed no porosity but were wrongly labelled as porosity images. This resulted in a poorly trained model and the model classified these images as non-porosity images. The images numbered 1, 5, 6 and 7 were correctly labelled but were wrongly classified. The images numbered 1 and 5 in Fig 13 were porosity images but classified as non-porosity images by the model. Whereas images numbered 6 and 7 were nonporosity images but were wrongly classified as porosity images by the model. The high number of wrongly predicted images by the model resulted in a poor performance with recall of only $54 \%$ and $90 \%$ accuracy. There are several reasons for this.

The porosity bubbles of size less than $0.2 \mathrm{~mm}$ designed in the CAD file were not visible to the naked eye on the powder bed images. The smallest identifiable spherical pore was $0.2 \mathrm{~mm}$ in diameter. Therefore the pores of size $0.1 \mathrm{~mm}, 0.05 \mathrm{~mm}$ and $0.02 \mathrm{~mm}$ were not formed in the test cylinder and did not appear on the in-process images as well. However, those images were wrongly classified as porosity images according to the CAD-assisted labelling approach.

For the second approach, we employed XCT analysis to verify the actual creation of pores in the test cylinders. The XCT analysis was used to see if pores smaller than $0.1 \mathrm{~mm}$ were formed in the parts. Pores smaller than $0.1 \mathrm{~mm}$ could not be seen in the XCT data, therefore the LPBF machine may not be capable of forming features of this size. As the pores in this study were designed into the CAD file, the way they are processed by the LPBF machine is different to how pores normally occur. The contour of each pore was scanned; this does not happen normally when porosity (lack of fusion or keyhole) forms. By scanning the boundary of 


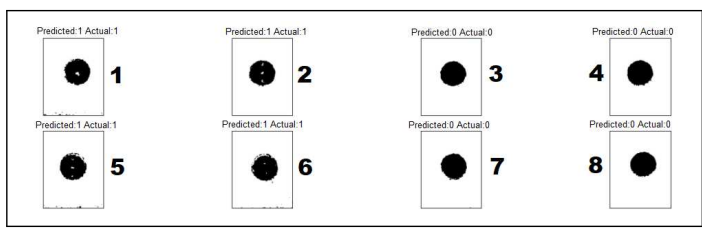

Fig. 14 Sample images of correct prediction for the XCTlabelled image model

the pore, the pore had a clear outer edge and was distinctly different to the melted material around it. Moreover, the images from the start and end of the circular porosity bubble were also not visible due to the changing cross sectional area of the pore. According to the XCT-assisted labelling approach, in-process images were categorised as porosity images only if their corresponding XCT images showed porosity. This resulted in a better labelled image data set and hence performed better. Some of the correctly predicted images following the XCT-assisted approach are shown in Fig 14. It can be observed that the model successfully distinguished between porosity and non-porosity images. The images numbered 1, 2, 5 and 6 in Fig 14 had porosity and were successfully identified by the model. Moreover, images numbered 3, 4, 7 and 8 had no porosity and were correctly classified as non-porosity images by the model. The model achieved a high accuracy of $97 \%$ with $85 \%$ recall on imbalanced data and $97 \%$ recall on balanced XCT-labelled images.

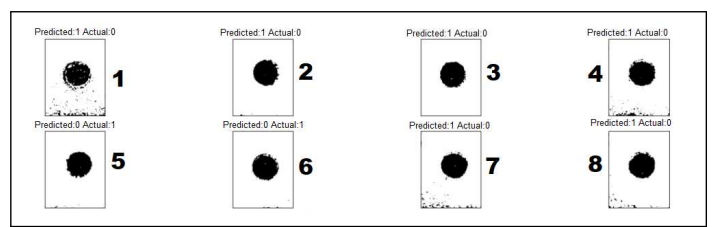

Fig. 15 Sample images of incorrect prediction of the XCT-labelled image model

The gas flow inside the build chamber sometimes blows spatter onto the recently melted section of the powder layer. This may appear on the image as porosity due to the image enhancement techniques employed in our experiments; but in reality it is not porosity. Fortunately, only a small number of images had this problem and they were less than ten in total. The images numbered 1, 4, 7 and 8 shown in Fig 15 were predicted as porosity images by the model but in reality they belonged to the non-porosity class. This is a rare phenomenon and the few miss-classified images in our experiments using the XCT-assisted labelling approach were mostly because of this problem.

The experiments revealed that labelling of the powder bed images from the printer could be improved significantly by using XCT images as a benchmark. This labelling technique is more reliable, effective, and yields better results. CADassisted labelling is straight forward and simple but impractical and generally only suitable for controlled proof-of-concept studies. We have successfully trained a deep CNN on powder bed images from an LPBF machine. The CNN model is capable of identifying defects as small as $0.2 \mathrm{~mm}$ sized pores on in-process images. In a similar study by [24], the smallest identifiable pore size by inprocess imaging was $0.6 \mathrm{~mm}$. However, Zhang et al. [38] trained a CNN model capable of identifying as small as $0.1 \mathrm{~mm}$ with an accuracy of $91.2 \%$. The model's performance depends on several factors. Among them, image standard and the pore size are the most crucial. We have successfully fabricated $3 \mathrm{D}$ metal cylinders with pore sizes in the range of $0.2 \mathrm{~mm}$ to $2 \mathrm{~mm}$. We not only captured the pores' creation on images but also successfully trained a deep neural network that is highly accurate in distinguishing the porosity and non-porosity images with an accuracy of $97 \%$.

\subsection{Next Steps}

In this study, synthetic seeding was used to directly form artefact defects as a demonstration of how ML can be applied to in-process imaging in LPBF. The size and shape of the seeded pores are very close to size and shape of natural porosity in LPBF. The limitation of this study is the spatial resolution of the camera set up with a pixel size equal to $0.2 \mathrm{~mm}$. A higher resolution camera, or a reduced distance between the camera and the build area could enable detection of smaller defects. Despite the hardware limitation of the current experiments, the study has successfully established the efficacy of CNNs in identifying the porosity from the in-process images of LPBF. It has also emphasised the significance of correct labelling of the LPBF images and fine tuning of the model to enhance the model's performance. The proposed CNN model is scalable and accurate. The model should be able to detect pore sizes smaller than $0.2 \mathrm{~mm}$ given high quality in-process 
images. Moreover, the current model could be used as a base model for future extension of LPBF defects detection. We have a strong belief that this model would provide a better base model network for transfer learning saving the need of training the model from start, data augmenting, and heavy computation on tuning hyper-parameters. This will likely provide a better starting point, significantly reduced the training time and computational resources needed for model's training. In further experiments, process parameters such as laser power, scan speed and hatch spacing could be varied to more closely simulate typical porosity in LPBF builds. Besides, future experiments will be used to study the transfer-ability of current model's learning and its ability to identify other porosity types such as lack of fusion and voids, as well as other LPBF micro-defects such as balling, surface deformation etc. The identification of porosity from powder bed images would enable a closed-loop real-time monitoring system. The advanced printers, such as EOS M290 by EOS, have real-time printing parameter changing capabilities. The proposed ML solution would enable practitioners to identify the defect and adjust printing parameters such as laser power, scan speed, etc., to avoid and remove defects. By automating defect detection from in-process imaging, the large data sets of layer images, collected from each build, could be summarised to highlight potential issues to the machine operator. This would significantly reduced the production cost as the 3D objects would require less postbuild quality assessment. Overall, this will enable a more robust supply chain and reduce the timeto-market of $3 \mathrm{D}$ objects.

\section{Conclusion}

This study has established the efficacy of ML models in detecting LPBF defects from powder bed images. The application of a deep neural network model to predict the porosity from inprocess images of LPBF was investigated. Besides data capture, labelling is the biggest challenge in the development of accurate ML models. Two labelling approaches were proposed, CAD-assisted labelling, and XCT-assisted labelling of in-process images of the test cylinders designed with seeded pores of sizes ranging from $0.02 \mathrm{~mm}$ to $2 \mathrm{~mm}$. Experiments revealed that the XCT information is a better benchmark for accurate data labelling. The CAD-assisted labelling was less reliable as the pores designed into the CAD file might not be created in the final test specimen. The deep CNN model trained on the CAD-assisted labelled data set achieved an accuracy of $90 \%$. However, the model failed to distinguish between porosity and non-porosity images in some cases and resulted in a high number of false-negatives due to the incorrect labelling of some of the images. In contrast, XCT-assisted labelling of the in-process images was reliable, accurate, effective, and produced better results. The deep CNN model distinguished the porosity images from non-porosity images with an accuracy of $97 \%$. The model successfully detected pores as small as $0.2 \mathrm{~mm}$ in size on in-process images. This is a big step towards porosity identification from in-process images. Moreover, we found that the balanced dataset resulted in a more generalised and unbiased learning/training of the model compared to imbalanced dataset. The experiments revealed that the balanced dataset significantly improved the model's precision from $89 \%$ to $97 \%$ and recall from $85 \%$ to $97 \%$ when compared to training on an imbalanced data set. The highly accurate predictability of porosity defect by proposed model will help in post-processing cost reduction. The early in-process real time detection of porosity will enable AM machine operator to adjust the printing parameters to avoid defects.

In future experiments, a better camera setting will help in capturing higher definition images. This will, in turn, enable the capture of smallersized porosity. To develop these findings further, objects would be designed to encourage defect formation during the LPBF process. Defects such as porosity, balling, lack of fusion, and surface deformation will be created naturally in test objects by altering different process parameters such as laser power, scan speed, and scan strategy. Moreover, current model's learning will be tested on new defects by transferring its learning/weights to new deep learning models.

Acknowledgments. The authors would like to acknowledge the support from Innovate UK for the project Defect Detection in Additive Manufacturing (105508). 


\section{Declarations}

The authors have no relevant financial or nonfinancial interests to disclose.

- Funding No funding was received to assist with the preparation of this manuscript.

- Conflict of interest/Competing interests (check journal-specific guidelines for which heading to use)

Not applicable.

- Ethics approval Not applicable.

- Consent to participate

Not applicable.

- Consent for publication Not applicable.

- Availability of data and materials Not applicable.

- Code availability Not applicable.

- Authors' contributions All the authors have contributed equally.

\section{References}

[1] Kellens, K., Baumers, M., Gutowski, T.G., Flanagan, W., Lifset, R., Duflou, J.R.: Environmental dimensions of additive manufacturing: Mapping application domains and their environmental implications. Journal of Industrial Ecology 21(S1), 49-68 (2017)

[2] Seabra, M., Azevedo, J., Araújo, A., Reis, L., Pinto, E., Alves, N., Santos, R., Mortágua, J.P.: Selective laser melting (slm) and topology optimization for lighter aerospace componentes. Procedia Structural Integrity 1, 289-296 (2016)

[3] Huang, R., Riddle, M., Graziano, D., Warren, J., Das, S., Nimbalkar, S., Cresko, J., Masanet, E.: Energy and emissions saving potential of additive manufacturing: the case of lightweight aircraft components. Journal of Cleaner Production 135, 1559-1570 (2016)

[4] Gebler, M., Uiterkamp, A.J.S., Visser, C.: A global sustainability perspective on $3 \mathrm{~d}$ printing technologies. Energy Policy 74, 158-167 (2014)
[5] Liu, Y., Yang, Y., Mai, S., Wang, D., Song, C.: Investigation into spatter behavior during selective laser melting of aisi 3161 stainless steel powder. Materials \& Design 87, 797-806 (2015)

[6] Levy, G.N., Schindel, R., Kruth, J.-P.: Rapid manufacturing and rapid tooling with layer manufacturing $(\mathrm{lm})$ technologies, state of the art and future perspectives. CIRP annals 52(2), 589-609 (2003)

[7] Koutiri, I., Pessard, E., Peyre, P., Amlou, O., De Terris, T.: Influence of slm process parameters on the surface finish, porosity rate and fatigue behavior of as-built inconel 625 parts. Journal of Materials Processing Technology 255, 536-546 (2018)

[8] Malekipour, E., El-Mounayri, H.: Common defects and contributing parameters in powder bed fusion am process and their classification for online monitoring and control: a review. The International Journal of Advanced Manufacturing Technology 95(14), 527-550 (2018)

[9] Romano, S., Brückner-Foit, A., Brandão, A., Gumpinger, J., Ghidini, T., Beretta, S.: Fatigue properties of alsi10mg obtained by additive manufacturing: Defect-based modelling and prediction of fatigue strength. Engineering Fracture Mechanics 187, 165189 (2018)

[10] Gordon, J.V., Narra, S.P., Cunningham, R.W., Liu, H., Chen, H., Suter, R.M., Beuth, J.L., Rollett, A.D.: Defect structure process maps for laser powder bed fusion additive manufacturing. Additive Manufacturing 36, 101552 (2020)

[11] Wang, J., Wu, W.J., Jing, W., Tan, X., Bi, G.J., Tor, S.B., Leong, K.F., Chua, C.K., Liu, E.: Improvement of densification and microstructure of astm a131 eh36 steel samples additively manufactured via selective laser melting with varying laser scanning speed and hatch spacing. Materials Science and Engineering: A 746, 300-313 (2019)

[12] Tucho, W.M., Lysne, V.H., Austbø, H., 
Sjolyst-Kverneland, A., Hansen, V.: Investigation of effects of process parameters on microstructure and hardness of slm manufactured ss3161. Journal of Alloys and Compounds 740, 910-925 (2018)

[13] Liverani, E., Toschi, S., Ceschini, L., Fortunato, A.: Effect of selective laser melting (slm) process parameters on microstructure and mechanical properties of $316 \mathrm{l}$ austenitic stainless steel. Journal of Materials Processing Technology 249, 255-263 (2017)

[14] Kasperovich, G., Haubrich, J., Gussone, J., Requena, G.: Correlation between porosity and processing parameters in tial6v4 produced by selective laser melting. Materials \& Design 105, 160-170 (2016)

[15] Shen, B., Li, H., Liu, S., Zou, J., Shen, S., Wang, Y., Zhang, T., Zhang, D., Chen, Y., Qi, H.: Influence of laser post-processing on pore evolution of ti-6al-4v alloy by laser powder bed fusion. Journal of Alloys and Compounds 818, 152845 (2020)

[16] Aboulkhair, N.T., Simonelli, M., Parry, L., Ashcroft, I., Tuck, C., Hague, R.: 3d printing of aluminium alloys: Additive manufacturing of aluminium alloys using selective laser melting. Progress in materials science 106, 100578 (2019)

[17] Aboulkhair, N.T., Everitt, N.M., Ashcroft, I., Tuck, C.: Reducing porosity in alsi10mg parts processed by selective laser melting. Additive Manufacturing 1, 77-86 (2014)

[18] Zhang, M., Sun, C.-N., Zhang, X., Goh, P.C., Wei, J., Hardacre, D., Li, H.: Fatigue and fracture behaviour of laser powder bed fusion stainless steel 3161: Influence of processing parameters. Materials Science and Engineering: A 703, 251-261 (2017)

[19] Gong, H., Rafi, K., Gu, H., Ram, G.J., Starr, T., Stucker, B.: Influence of defects on mechanical properties of ti-6al-4 $\mathrm{v}$ components produced by selective laser melting and electron beam melting. Materials \& Design 86, 545-554 (2015)
[20] du Plessis, A.: Effects of process parameters on porosity in laser powder bed fusion revealed by x-ray tomography. Additive Manufacturing 30, 100871 (2019)

[21] Choo, H., Sham, K.-L., Bohling, J., Ngo, A., Xiao, X., Ren, Y., Depond, P.J., Matthews, M.J., Garlea, E.: Effect of laser power on defect, texture, and microstructure of a laser powder bed fusion processed 3161 stainless steel. Materials \& Design 164, 107534 (2019)

[22] Leung, C.L.A., Marussi, S., Towrie, M., Atwood, R.C., Withers, P.J., Lee, P.D.: The effect of powder oxidation on defect formation in laser additive manufacturing. Acta Materialia 166, 294-305 (2019)

[23] Mireles, J., Terrazas, C., Gaytan, S.M., Roberson, D.A., Wicker, R.B.: Closedloop automatic feedback control in electron beam melting. The International Journal of Advanced Manufacturing Technology 78(58), 1193-1199 (2015)

[24] Mireles, J., Ridwan, S., Morton, P.A., Hinojos, A., Wicker, R.B.: Analysis and correction of defects within parts fabricated using powder bed fusion technology. Surface Topography: Metrology and Properties 3(3), 034002 (2015)

[25] Gobert, C., Reutzel, E.W., Petrich, J., Nassar, A.R., Phoha, S.: Application of supervised machine learning for defect detection during metallic powder bed fusion additive manufacturing using high resolution imaging. Additive Manufacturing 21, 517-528 (2018)

[26] Wits, W.W., Carmignato, S., Zanini, F., Vaneker, T.H.: Porosity testing methods for the quality assessment of selective laser melted parts. CIRP annals 65(1), 201-204 (2016)

[27] Okaro, I.A., Jayasinghe, S., Sutcliffe, C., Black, K., Paoletti, P., Green, P.L.: Automatic fault detection for laser powderbed fusion using semi-supervised machine learning. Additive Manufacturing 27, 42-53 (2019) 
[28] Everton, S.K., Hirsch, M., Stravroulakis, P., Leach, R.K., Clare, A.T.: Review of in-situ process monitoring and in-situ metrology for metal additive manufacturing. Materials \& Design 95, 431-445 (2016)

[29] Yuan, B., Giera, B., Guss, G., Matthews, I., Mcmains, S.: Semi-supervised convolutional neural networks for in-situ video monitoring of selective laser melting. In: 2019 IEEE Winter Conference on Applications of Computer Vision (WACV), pp. 744-753 (2019). IEEE

[30] Qi, X., Chen, G., Li, Y., Cheng, X., Li, C.: Applying neural-network-based machine learning to additive manufacturing: Current applications, challenges, and future perspectives. Engineering (2019)

[31] Quinlan, J.R.: Induction of decision trees. Machine learning 1(1), 81-106 (1986)

[32] Breiman, L.: Random forests. Machine learning 45(1), 5-32 (2001)

[33] Noble, W.S.: What is a support vector machine? Nature biotechnology 24(12), 1565-1567 (2006)

[34] Chouiekh, A., EL Haj, E.H.I.: Convnets for fraud detection analysis. Procedia Computer Science 127, 133-138 (2018). https: //doi.org/10.1016/j.procs.2018.01.107. PROCEEDINGS OF THE FIRST INTERNATIONAL CONFERENCE ON INTELLIGENT COMPUTING IN DATA SCIENCES, ICDS2017

[35] Pan, S.J., Yang, Q.: A survey on transfer learning. IEEE Transactions on knowledge and data engineering 22(10), 1345-1359 (2009)

[36] Zamir, A.R., Sax, A., Shen, W., Guibas, L.J., Malik, J., Savarese, S.: Taskonomy: Disentangling task transfer learning. In: Proceedings of the IEEE Conference on Computer Vision and Pattern Recognition, pp. 37123722 (2018)

[37] Kwon, O., Kim, H.G., Ham, M.J., Kim, W., Kim, G.-H., Cho, J.-H., Kim, N.I., Kim, K.:
A deep neural network for classification of melt-pool images in metal additive manufacturing. Journal of Intelligent Manufacturing, 1-12 (2018)

[38] Zhang, B., Liu, S., Shin, Y.C.: In-process monitoring of porosity during laser additive manufacturing process. Additive Manufacturing 28, 497-505 (2019)

[39] Li, X., Jia, X., Yang, Q., Lee, J.: Quality analysis in metal additive manufacturing with deep learning. Journal of Intelligent Manufacturing 31(8), 2003-2017 (2020)

[40] Aminzadeh, M., Kurfess, T.R.: Online quality inspection using bayesian classification in powder-bed additive manufacturing from high-resolution visual camera images. Journal of Intelligent Manufacturing 30(6), 25052523 (2019)

[41] Grasso, M., Demir, A.G., Previtali, B., Colosimo, B.M.: In situ monitoring of selective laser melting of zinc powder via infrared imaging of the process plume. Robotics and Computer Integrated Manufacturing 49, 229-239 (2018)

[42] Scime, L., Beuth, J.: Using machine learning to identify in-situ melt pool signatures indicative of flaw formation in a laser powder bed fusion additive manufacturing process. Additive Manufacturing 25, 151-165 (2019)

[43] Scime, L., Siddel, D., Baird, S., Paquit, V.: Layer-wise anomaly detection and classification for powder bed additive manufacturing processes: A machine-agnostic algorithm for real-time pixel-wise semantic segmentation. Additive Manufacturing 36, 101453 (2020)

[44] Bartlett, J.L., Heim, F.M., Murty, Y.V., Li, $\mathrm{X}$.: In situ defect detection in selective laser melting via full-field infrared thermography. Additive Manufacturing 24, 595-605 (2018)

[45] Ye, D., Hsi Fuh, J.Y., Zhang, Y., Hong, G.S., Zhu, K.: In situ monitoring of selective laser melting using plume and spatter signatures by deep belief networks. ISA Transactions $\mathbf{8 1}$, 96-104 (2018) 
[46] Caggiano, A., Zhang, J., Alfieri, V., Caiazzo, F., Gao, R., Teti, R.: Machine learning-based image processing for on-line defect recognition in additive manufacturing. CIRP Annals - Manufacturing Technology 68(1), 451-454 (2019)

[47] Scime, L., Beuth, J.: A multi-scale convolutional neural network for autonomous anomaly detection and classification in a laser powder bed fusion additive manufacturing process. Additive Manufacturing 24, 273-286 (2018)

[48] Scime, L., Beuth, J.: Anomaly detection and classification in a laser powder bed additive manufacturing process using a trained computer vision algorithm. Additive Manufacturing 19, 114-126 (2018)

[49] Otsu, N.: A threshold selection method from gray-level histograms. IEEE transactions on systems, man, and cybernetics 9(1), 62-66 (1979)

[50] Havaei, M., Davy, A., Warde-Farley, D., Biard, A., Courville, A., Bengio, Y., Pal, C., Jodoin, P.-M., Larochelle, H.: Brain tumor segmentation with deep neural networks. Medical image analysis 35, 18-31 (2017)

[51] Pereira, S., Pinto, A., Alves, V., Silva, C.A.: Brain tumor segmentation using convolutional neural networks in mri images. IEEE transactions on medical imaging 35(5), 12401251 (2016)

[52] Kamnitsas, K., Ledig, C., Newcombe, V.F., Simpson, J.P., Kane, A.D., Menon, D.K., Rueckert, D., Glocker, B.: Efficient multiscale 3d cnn with fully connected crf for accurate brain lesion segmentation. Medical image analysis 36, 61-78 (2017)

[53] Li, R., Feng, F., Ahmad, I., Wang, X.: Retrieving real world clothing images via multi-weight deep convolutional neural networks. Cluster Computing 22(3), 7123-7134 (2019)
[54] Polz, J., Chwala, C., Graf, M., Kunstmann, H.: Rain event detection in commercial microwave link attenuation data using convolutional neural networks. Atmospheric Measurement Techniques 13(7), 3835-3853 (2020)

[55] Kingma, D.P., Ba, J.: Adam: A method for stochastic optimization. arXiv preprint arXiv:1412.6980 (2014)

[56] Li, L., Jamieson, K., DeSalvo, G., Rostamizadeh, A., Talwalkar, A.: Hyperband: A novel bandit-based approach to hyperparameter optimization. The Journal of Machine Learning Research 18(1), 6765-6816 (2017)

[57] Tajbakhsh, N., Shin, J.Y., Gurudu, S.R., Hurst, R.T., Kendall, C.B., Gotway, M.B., Liang, J.: Convolutional neural networks for medical image analysis: Full training or fine tuning? IEEE transactions on medical imaging 35(5), 1299-1312 (2016)

[58] Thenmozhi, K., Reddy, U.S.: Crop pest classification based on deep convolutional neural network and transfer learning. Computers and Electronics in Agriculture 164, 104906 (2019) 